\title{
Tunable Fano Resonance and Enhanced Sensing in Terahertz Metamaterial
}

\author{
Yun Wang, Shengyao Jia and Jianyuan Qin* \\ Center for Terahertz Research, China Jiliang University, Hangzhou, China
}

\section{OPEN ACCESS}

Edited by: Longqing Cong, University of Pennsylvania,

United States

Reviewed by:

Yogesh Kumar Srivastava, Nanyang Technological University, Singapore

Dejun Liu,

Shanghai Normal University, China

*Correspondence: Jianyuan Qin jyqin@cjlu.edu.cn

Specialty section: This article was submitted to Optics and Photonics,

a section of the journal

Frontiers in Physics.

Received: 11 September 2020 Accepted: 17 November 2020

Published: 08 January 2021

Citation:

Wang Y, Jia S and Qin J (2021) Tunable Fano Resonance and Enhanced Sensing in Terahertz Metamaterial.

Front. Phys. 8:605125. doi: 10.3389/fphy.2020.605125
Fano resonances in metamaterial are important due to their low-loss subradiant behavior that allows excitation of high quality $(Q)$ factor resonances extending from the microwave to the optical bands. Fano resonances have recently showed their great potential in the areas of modulation, filtering, and sensing for their extremely narrow linewidths. However, the Fano resonances in a metamaterial system arise from the interaction of all that form the structure, limiting the tunability of the resonances. Besides, sensing trace analytes using Fano resonances are still challenging. In the present work, we demonstrate the excitation of Fano resonances in metamaterial consisting of a period array of two concentric doublesplit-ring resonators with symmetry breaking (position asymmetry and gaps asymmetry). The tunability and sensing of Fano resonances are both studied in detail. Introducing position asymmetry in the metamaterial leads to one Fano resonance located at $0.50 \mathrm{THz}$, while introducing gaps asymmetry results in two Fano resonances located at $0.35 \mathrm{THz}$ and $0.50 \mathrm{THz}$. The transmittance, position, and linewidth of the three Fano resonances can be easily tuned by varying the asymmetry deviations. The $Q$ factor and figure of merit (FoM) of Fano resonances with different asymmetry deviations are calculated for performance optimization. The Fano resonances having the highest FoM are used for the sensing of analytes at different refractive indices, and the Fano resonance performing the best in refractive index sensing is further applied to detect the analyte thickness. The results demonstrate that the tunable Fano resonances show tremendous potential in sensing applications, offering an approach to engineering highly efficient modulators and sensors.

Keywords: terahertz, metamaterial, Fano resonance, tunable, sensing

\section{INTRODUCTION}

Terahertz (THz) waves ranging from 0.1 to $10 \mathrm{THz}$ show huge potential for application in communication [1], imaging [2, 3], biomedicine [4-8], and chemistry [9-12]. The great prospects of $\mathrm{THz}$ technology inevitably stimulate further research on the cost-effective devices to manipulate the emission, propagation, modulation, and sensing of $\mathrm{THz}$ waves. Metamaterial (MM) devices comprising artificially designed structures have been widely applied in $\mathrm{THz}$ technology, which has greatly changed conventional approaches for manipulating $\mathrm{THz}$ waves. MM structures possess unique electromagnetic properties that are different from natural materials; such extraordinary features provide them the ability to effectively control the $\mathrm{THz}$ waves. The electromagnetic properties of $\mathrm{MM}$ depend on the layout, orientation, and geometry of subwavelength resonators in each unit cell, as well as the coupling between the unit cells. These findings have facilitated exquisite research and acquired exciting advancements such as superlenses 
[13], communication [14], absorbers [15-18], cloaks [19], and sensors [20-25] in the field of $\mathrm{THz}$ waves. Split-ring resonator (SRR) array on a high transmittance substrate is the most popular MM structure in the aforementioned application area. SRR-based MM can exhibit inductive-capacitive resonance as the polarization of the incident $\mathrm{THz}$ waves is parallel to the gap of SRR. However, the dipole resonance can be excited when the polarization of incident $\mathrm{THz}$ waves is perpendicular to the gap of SRR. Both resonances have a relatively broad linewidth as well as a quality $(Q)$ factor below 10 [26]. The low $Q$ factor of these resonances is due to the radiative and nonradiative loss mechanisms in the MM [27]. At THz frequencies, the Drude metals, such as gold, copper, and aluminum, have extremely high conductivity that eliminates most of the nonradiative losses [28]. Thus, the radiative losses play a dominant role in the THz MM structure. It has been found that the dominant radiative losses can be controlled by optimizing the geometry of the metallic subwavelength structure to excite sharp asymmetric resonance with high $Q$ factor. Such resonance is termed as Fano resonance and exhibits a very high $Q$ factor even up to 75 [29]. Fano resonance arises from the plasmonic hybridization between narrow discrete resonance (dark mode) and a broad spectral line or continuum (bright mode). It is found that Fano resonance shows asymmetric and sharp spectral lines with narrow linewidth and has strongly confined electromagnetic fields. Because of these excellent features, Fano resonances have great potential to be applied to the design of $\mathrm{THz}$ high $Q$ devices. An ultrahigh $Q$ factor of 227 could be observed for the lowest asymmetry case in an asymmetric SRR-based MM structure [30]. However, the resonance intensity in this structure was extremely weak, and thus, the figure of merit (FoM) is lower than 47. For practical applications, a sharp resonance feature as well as strong resonance intensity is typically desired. Therefore, a SRR-based MM with Fano resonance having both ultrahigh $Q$ factor and high FoM has been proposed [31]. The $Q$ factor up to 394.5 and a high FoM up to 371.9 under the normal incidence of linear polarized light could be achieved by the displacement asymmetry. Among the emergent functionalities, the tunability of Fano resonance in a MM structure plays a crucial role in controlling the resonance characteristics including the position and the intensity of the resonance. Nonetheless, the accurate control of Fano resonances in coupling resonators is still difficult to achieve, which greatly hinders the development of Fano resonance-based $\mathrm{MM}$ devices for diverse $\mathrm{THz}$ applications.

Numerous molecular vibrations including molecular rotations, low frequency bond vibrations, and crystalline phonon vibrations are all present in $\mathrm{THz}$ region, enabling the identification and detection of molecules using $\mathrm{THz}$ technology. Besides, due to the advantages of low energy and high penetration, the performance of $\mathrm{THz}$ waves is excellent in nondestructive testing. Recently, $\mathrm{THz}$ technology has showed great potential in the fields of biomedicine and chemistry. However, the sensing of analytes with high sensitivity is still challenging. The strong electric field of Fano resonances can greatly enhance the interaction between the light and matters, which leads to high sensitivity in sensing applications. Therefore, Fano resonance-based MM has been used in THz technology for improving the detection sensitivity. The low-loss Fano resonances with ultra-sharp feature enable measuring the minute spectral shift due to the small change in the refractive index of the surrounding media. A simulated sensitivity level of $5.7 \times 10^{4} \mathrm{~nm} / \mathrm{RIU}$ was achieved, and the sensitivity could be further enhanced by using thinner substrates [32]. The combination of Fano resonance and microfluidic chip could realize the detection of trace liquid samples with a concentration of $1.4 \mathrm{fmol}$ in a volume of $128 \mathrm{pL}$. Compared with the symmetric structure, the sensitivity of the asymmetric structure based on Fano resonance was increased by an order of magnitude [33]. In these studies, it is noted that MM working at $\mathrm{THz}$ frequencies could be easily fabricated by using the photolithograph process, which is of low cost and low requirement for accuracy compared with the fabrication of the MM working at other frequencies. Therefore, the research on the $\mathrm{THz}$ sensing using Fano resonance-based MM will provide an effective approach for highly sensitive sensing and promote the application and development of $\mathrm{THz}$ technology. In our present study, the tunability and application of the Fano resonances in MM structure arising due to the symmetric breaking are demonstrated. Different from the previous reports [32, 34, 35], our MM structure comprises a period array of two concentric SRRs. Introducing position asymmetry and gaps asymmetry can change the coupling between the two concentric SRRs and lead to the excitation of three Fano resonances (multiple Fano resonance). These resonances can be tuned by varying the asymmetry deviation. The $Q$ factor and FoM are calculated to estimate the performances of the asymmetric MM structures at different asymmetry deviations. The best-performing Fano resonances with the highest FoM are used for sensing application by coating an analyte layer on the surface of MM structure. The sensitivities of Fano resonances are studied to evaluate the ability of these resonances in the detection of refractive index. The sensing of analyte with different thicknesses is also investigated to demonstrate the capability of Fano resonance for thickness sensing.

\section{STRUCTURE DESIGN}

Figure 1A shows the schematic of the MM structure being excited by normally incident $\mathrm{THz}$ wave. The coordinates of electric field $(E)$, magnetic field $(H)$, and wave vector $(k)$ of incident $\mathrm{THz}$ wave are also indicated. The MM structure consists of a periodic array of two copper concentric SRRs, the inner SRR and the outer SRR, on a high-resistivity silicon substrate. Figure $\mathbf{1 B}$ shows the unit cell of the MM-1 structure on the xy plane, which is symmetric along $x$-axis and $y$-axis. Structural symmetry of the unit cell was broken forming asymmetric MM to study the coupling between these two concentric SRRs. For MM-2, see Figure 1C; the position of inner SRR is off the center, and it moves rightwards by $d=2.4 \mu \mathrm{m}$ along $x$-axis to form a position asymmetric MM structure. Meanwhile, for MM-3, the lower gaps of the two SRRs deviate from $y$-axis, and they both move rightwards by $s=6 \mu \mathrm{m}$ along $x$-axis, creating a gap asymmetric MM structure. All three structures have the same dimension 

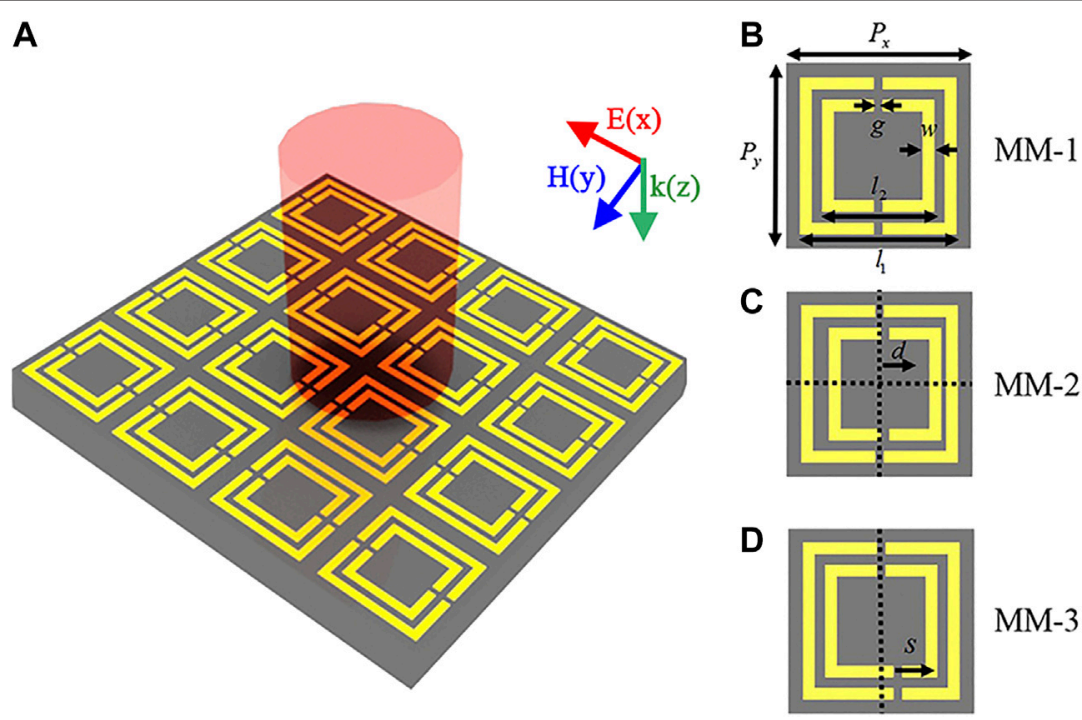

FIGURE 1 | (A) Three-dimensional schematic of the MM structure being excited by a normally incident THz pulse. Two-dimensional schematic of unit cell for (B) symmetric MM structure (MM-1), (C) position asymmetric MM structure (MM-2), and (D) gaps asymmetric MM structure (MM-3) with all dimensions at a micron scale. In (C), the position of inner SRR moves rightwards by $d$ along $x$-axis. In (D), the lower gaps of two SRRs move rightwards by $s$ along $x$-axis. All three structures have the same dimension parameters: $I_{1}=84 \mu \mathrm{m}, I_{2}=60 \mu \mathrm{m}, g=3 \mu \mathrm{m}, w=6 \mu \mathrm{m}$, and $P_{\mathrm{x}}=P_{\mathrm{y}}=100 \mu \mathrm{m}$. The thicknesses of metallic layer and substrate layer are 0.2 and $40 \mu \mathrm{m}$, respectively.

parameters: $l_{1}=84 \mu \mathrm{m}, l_{2}=60 \mu \mathrm{m}, w=6 \mu \mathrm{m}, g=3 \mu \mathrm{m}, P_{\mathrm{x}}=$ $P_{\mathrm{y}}=100 \mu \mathrm{m}$, the copper thickness $t_{1}=0.2 \mu \mathrm{m}$, and the silicon substrate thickness $t_{2}=500 \mu \mathrm{m}$. The simulations were carried out using the commercially available CST Microwave Studio. The frequency domain solver was adopted with the periodic boundary conditions in the $x$ direction and $y$ direction, while the $z$ direction was open to free space. The $\mathrm{THz}$ wave with an electric field linearly polarized along $x$-axis was vertically incident on the surface of MM structure. The conductivity of copper was set to $\sigma_{\mathrm{dc}}=5.76 \times 10^{7} \mathrm{~S} / \mathrm{m}$, and the permittivity of silicon was $\varepsilon=11.9$.

The tuning of the Fano resonances in MM structure arising from the symmetric breaking was investigated by varying the position asymmetric deviation $d$ and gap asymmetric deviation $s$. The transmittance spectra and the electromagnetic field distribution at resonances for different asymmetric deviations were simulated to analyze the coupling between the concentric SRRs within the unit cell. The $Q$ factor and the FoM were also calculated. In order to confirm the sensing ability of the tunable Fano resonances, the detection of refractive index, as well as the sensing of analyte thickness, was investigated, and the sensitivities were calculated to evaluate the sensing performance.

\section{RESULTS AND DISCUSSIONS}

The transmittance spectra of MM-1, MM-2, and MM-3 are shown in Figure 2A. MM-1 has two obvious transmission dips located at $0.40 \mathrm{THz}$ and $0.68 \mathrm{THz}$ (black line). For the dip at $0.40 \mathrm{THz}$, it can be seen from Figure $\mathbf{2 B}$ that the surface currents are mainly distributed in the left arm and right arm of the outer SRR, and they run parallel in these two arms. The electric field is mainly located in the upper and lower components of the outer SRR in this case. As for $0.68 \mathrm{THz}$, see Figure 2C, the parallel surface currents are mainly located in the left arm and right arm of the inner SRR. In this case, the electric field is concentrated in the upper and lower components of the inner SRR. Therefore, these two resonances are classified as dipole resonance and labeled as $\mathrm{DD}_{1}$ and $\mathrm{DD}_{2}$. After introducing the position asymmetry $(d=2.4 \mu \mathrm{m})$, one more transmission dip at $0.50 \mathrm{THz}$ appears for MM-2 besides $\mathrm{DD}_{1}$ and $\mathrm{DD}_{2}$ (red line). As shown in Figure 2D, for $0.50 \mathrm{THz}$, the coupling of two SRRs leads to the concentration of electric field in the two gaps of the inner SRR, and the surface currents in the two SRRs are antiparallel, indicating that this resonance is attributed to the Fano mode. Thus, the resonance of $0.50 \mathrm{THz}$ in MM-2 is termed as Fano resonance and marked as $\mathrm{FD}_{1}$. By introducing the gap asymmetry $(s=6 \mu \mathrm{m})$, there are two more transmission dips at $0.35 \mathrm{THz}$ and $0.50 \mathrm{THz}$ for MM-3 (blue line). Figure 2E shows that the electric field is concentrated in the gaps of the outer SRR at the resonance of $0.35 \mathrm{THz}$. The antiparallel surface currents in this case are mainly distributed in the left arm and right arm of the outer SRR, and the currents in the left arm are much stronger than those in the right arm. For the dip of $0.5 \mathrm{THz}$, similar to $\mathrm{FD}_{1}$, the coupling of the two SRRs causes antiparallel surface currents in the two SRRs with stronger currents distributed in their left arms and results in strong electric field located in the gaps of the inner SRR; see Figure 2F. The distributions of surface currents and electric field indicate that the excitations of dips in MM-3 at $0.35 \mathrm{THz}$ and $0.50 \mathrm{THz}$ origin from the Fano modes. Therefore, they are termed as Fano resonances and marked as $\mathrm{FD}_{2}$ and $\mathrm{FD}_{3}$. Note that $\mathrm{DD}_{1}$ and $\mathrm{DD}_{2}$ appear in all three structures, and their resonant frequencies remain almost unchanged. 

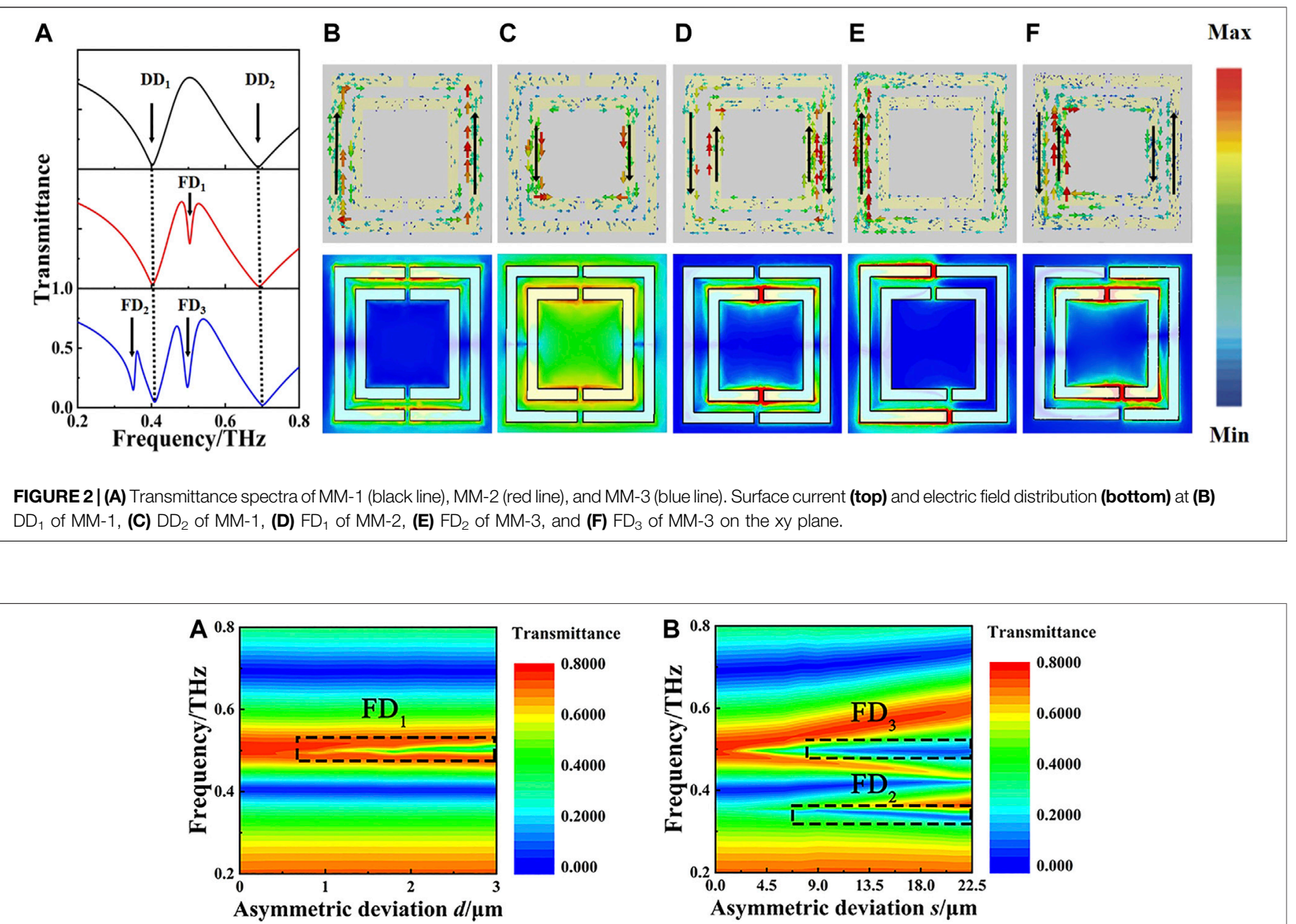

FIGURE 3 | Two-dimensional diagram of transmittance as a function of frequency and asymmetric deviation for (A) FD 1 of $\mathrm{MM}-2$ and $(\mathbf{B}) \mathrm{FD}_{2}$ and $\mathrm{FD}_{3}$ of $\mathrm{MM}-3$.

These Fano resonances $\left(\mathrm{FD}_{1}, \mathrm{FD}_{2}\right.$, and $\left.\mathrm{FD}_{3}\right)$ arise due to the symmetry breaking, and they can be tuned by varying the asymmetric deviation. To study the dependence of tunable resonances on the asymmetric deviation, a fine simulation for $\mathrm{THz}$ transmittance of MM-2 as a function of frequency and asymmetric deviation $d$ and of MM-3 as a function of frequency and asymmetric deviation $s$ was performed, and the results are displayed in Figure 3. The asymmetric deviation $d$ increases from 0.6 to $3 \mu \mathrm{m}$ with a simulated step of $0.25 \mu \mathrm{m}$, while the asymmetric deviation $s$ increases from 3 to $22.5 \mu \mathrm{m}$ with a simulated step of $0.50 \mu \mathrm{m}$. It is found that as the asymmetric deviation $d$ increases gradually, $\mathrm{FD}_{1}$ of $\mathrm{MM}-2$ arises, and its resonance position remains almost unchanged, as shown in Figure 3A. However, the transmittance of $\mathrm{FD}_{1}$ slightly decreases, and its linewidth is becoming wider with $d$ increasing. For $\mathrm{MM}-3, \mathrm{FD}_{2}$ and $\mathrm{FD}_{3}$ appear with decreasing transmittance when the asymmetric deviation $s$ increases, see Figure 3B. Continuing to increasing $s, \mathrm{FD}_{2}$ shows a slight redshift. In this case, however, the position of $\mathrm{FD}_{3}$ is almost fixed at $0.5 \mathrm{THz}$, and its linewidth is getting wider. These results demonstrate that the resonance characteristics of these resonances, including the transmittance, position, and linewidth, can be tuned by changing the asymmetric deviations; such tunability has potential applications in the design of filters, modulators, switches, and sensors devices.

Next, the performances of the two asymmetric MM structures (MM-2 and MM-3) vs. the asymmetric deviations $d$ and $s$ are evaluated in terms of $Q$ factor and FoM. Here, the $Q$ factor is defined as $Q=f_{0} /$ FWHM, where $f_{0}$ is the frequency of the resonance and FWHM is the full width at half maximum. The FoM is defined as FoM $=Q \times \Delta I$, where $\Delta I$ is the resonance intensity [28]. Figure 4 depicts the responses of $Q$ factor and FoM when the asymmetric deviation changes. The black spheres and red spheres depict the $Q$ factor and FoM at different asymmetric deviations, respectively. It can be seen from Figure $4 \mathrm{~A}$ that the $Q$ factor of $\mathrm{FD}_{1}$ is 93 when the asymmetric deviation $d$ is $0.6 \mu \mathrm{m}$. Then, it decreases as the asymmetric deviation $d$ increases. In this case, the FoM increases with the increase of the asymmetric deviation $d$ and reaches the saturated value of 13 when $d=$ $1.8 \mu \mathrm{m}$. As for $\mathrm{FD}_{2}$, see Figure $4 \mathrm{~B}$; the change in $Q$ factor is similar to that of $\mathrm{FD}_{1}$. When $s=3 \mu \mathrm{m}$, the maximum of $Q$ factor is about 78, and then it keeps decreasing as the asymmetric deviation $s$ increases, while the FoM grows rapidly to a maximum of 6 at $s=9 \mu \mathrm{m}$ and decreases slowly after that. For 

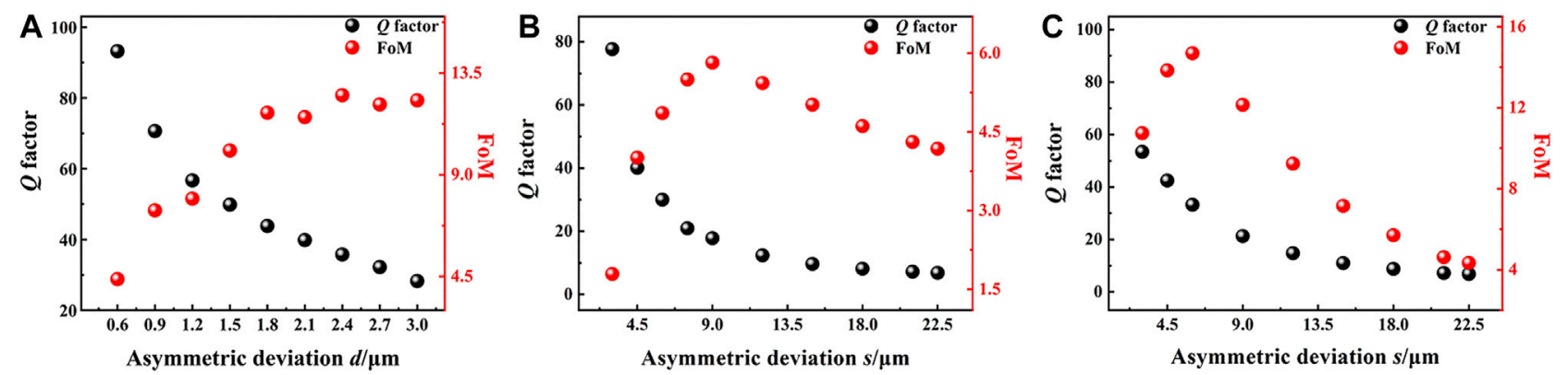

FIGURE 4 | Q factor (black spheres) and FoM (red spheres) vs. asymmetric parameter for (A) FD 1 in $M$ M-2, (B) FD in MM-3, and (C) FD3 in MM-3.
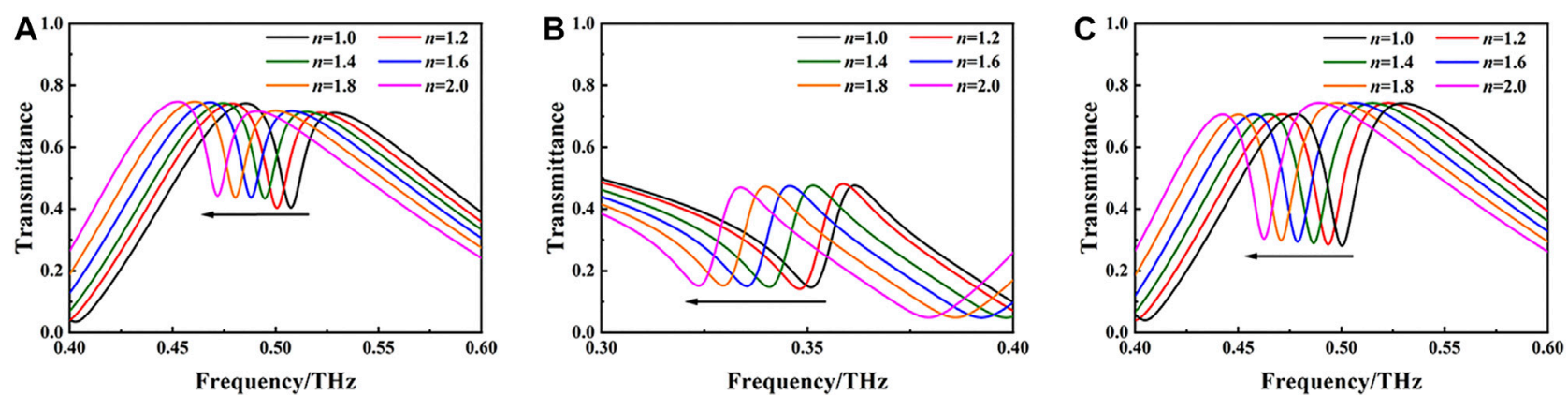

FIGURE 5 | Transmittance in the vicinity of (A) $\mathrm{FD}_{1}$, (B) $\mathrm{FD}_{2}$, and $\mathbf{( C )} \mathrm{FD}_{3}$ for the asymmetric MM structures coated with a different refractive index of analyte. The thickness of analyte is set to $4 \mu \mathrm{m}$, the asymmetric deviation $d$ for $\mathrm{FD}_{1}$ is $2.4 \mu \mathrm{m}$, and the asymmetric deviations $s$ for $\mathrm{FD}_{2}$ and $\mathrm{FD}_{3}$ are 9 and $6 \mu \mathrm{m}$, respectively.

$\mathrm{FD}_{3}$, the $Q$ factor and FoM both show similar curve shapes to those of $\mathrm{FD}_{2}$, as shown in Figure 4C. The $Q$ factor has a maximum of 82 at $s=3 \mu \mathrm{m}$, and the FoM peaks its maximum of 15 at about $s=6 \mu \mathrm{m}$. The higher the FoM is, the better the resonance performs. Therefore, the best performances of $\mathrm{FD}_{1}, \mathrm{FD}_{2}$, and $\mathrm{FD}_{3}$ are at the asymmetric deviation of $2.4,9$, and $6 \mu \mathrm{m}$, respectively.

In sensing applications, a sharp resonance feature and strong resonance intensity are both typically desired to detect a minute frequency shift that arises due to the small change in analyte. As mentioned earlier, the FoM that describes the $Q$ factor and the resonance intensity peaks at $d=2.4 \mu \mathrm{m}$ for $\mathrm{FD}_{1}, s=9 \mu \mathrm{m}$ for $\mathrm{FD}_{2}$, and $s=6 \mu \mathrm{m}$ for $\mathrm{FD}_{3}$, and these asymmetric deviations are chosen for asymmetric MM structures to investigate the sensing performances of these tunable resonances. Figure 5 shows the transmittance at around $\mathrm{FD}_{1}, \mathrm{FD}_{2}$, and $\mathrm{FD}_{3}$ for the asymmetric MM structures coated with different refractive index $(n)$ of analyte. The thickness of analyte is fixed at $4 \mu \mathrm{m}$. It is found that all three dips show a red-shift and slight increasing transmittance as the refractive index of analyte increases. When the refractive index increases from 1.0 to $1.2, \mathrm{FD}_{1}$ shifts with a value of $6.87 \mathrm{GHz}$. In this case, the frequency shifts for $\mathrm{FD}_{2}$ and $\mathrm{FD}_{3}$ are 3.86 and $7.03 \mathrm{GHz}$, respectively. With a further increase in the refractive index to 2.0, $\mathrm{FD}_{1}, \mathrm{FD}_{2}$, and $\mathrm{FD}_{3}$ have a frequency shift of $34.3,27.9$, and $38.4 \mathrm{GHz}$, respectively. For a more clear display of the relationship between the frequency shift and the refractive index, the frequency shifts vs. refractive index for the all three resonances and the corresponding linear fittings (dashed lines) are plotted in
Figure 6A. The linear fitting of $\mathrm{FD}_{3}$ has the biggest slope, followed by $\mathrm{FD}_{1}$, while $\mathrm{FD}_{2}$ has the smallest slope. The sensitivity is applied to evaluate the sensing performances of these resonances. Here, the sensitivity is defined as $S=\Delta f / \Delta n$, where $\Delta f$ is the frequency shift and $\Delta n$ is the change in refractive index of analyte. In this way, the sensitivities of $\mathrm{FD}_{1}, \mathrm{FD}_{2}$, and $\mathrm{FD}_{3}$ are calculated to be $34.3 \mathrm{GHz} / \mathrm{RIU}, 27.9 \mathrm{GHz} / \mathrm{RIU}$, and $38.4 \mathrm{GHz} / \mathrm{RIU}$, respectively. Thus, $\mathrm{FD}_{3}$ performs the best in the sensing of refractive index. The difference in sensitivity of the three resonances can be attributed to the difference in electric field intensity located at the gaps [36]. Stronger electric field will provide a stronger interaction between the electromagnetic wave and the analyte, which results in higher sensitivity [37]. Further study of the thickness sensing using $\mathrm{FD}_{3}$ was also conducted, and the results are shown in Figure 6B. The refractive index of analyte is 1.6, while the analyte thickness is changed from $0 \mu \mathrm{m}$ (without analyte) to $16 \mu \mathrm{m}$. The results show that the frequency shift of $\mathrm{FD}_{3}$ sharply increases when the analyte thickness increases from 0 to $5 \mu \mathrm{m}$, and it increases slowly as the analyte thickness varies from 5 to $12.5 \mu \mathrm{m}$. When the thickness of the analyte is $12.5 \mu \mathrm{m}$, the frequency shift reaches a saturated value of $16 \mathrm{GHz}$. These results show that the proposed structure is more suitable for sensing the thickness of thin layer analyte.

\section{CONCLUSION}

In summary, we have demonstrated that Fano resonances can be excited by introducing asymmetry (position asymmetry and gaps 

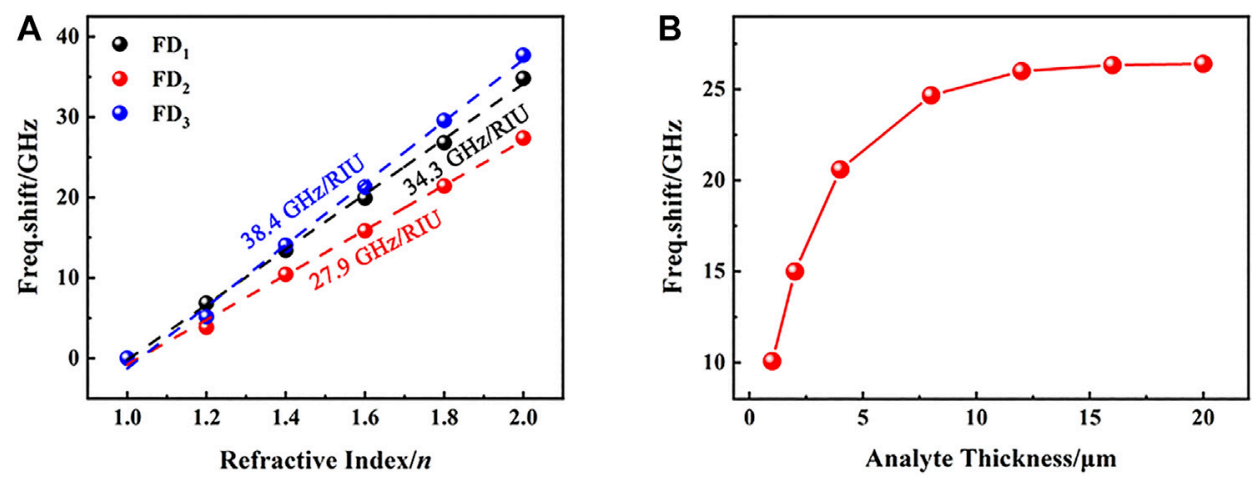

FIGURE 6 | (A) Frequency shift of $\mathrm{FD}_{1}$ (black spheres), $\mathrm{FD}_{2}$ (red spheres), and $\mathrm{FD}_{3}$ (blue spheres) vs. refractive index. (B) Frequency shift of FD 3 vs. analyte thickness.

asymmetry) in the $\mathrm{MM}$ formed by a period array of two concentric SRRs. One Fano resonance $\left(\mathrm{FD}_{1}\right)$ appears when the inner SRR is off the center, and two resonances $\left(\mathrm{FD}_{2}\right.$ and $\left.\mathrm{FD}_{3}\right)$ can be observed as the lower gaps of the two SRRs deviate from the $y$-axis. These phenomena are attributed to the coupling and the interaction between the two concentric SRRs. Variations in the asymmetric deviations can modulate the Fano resonances. The FoMs for $\mathrm{FD}_{1}, \mathrm{FD}_{2}$, and $\mathrm{FD}_{3}$ can reach 13,6 , and 15 , respectively. The sensitivities of $\mathrm{FD}_{1}, \mathrm{FD}_{2}$, and $\mathrm{FD}_{3}$ used for refractive index sensing are $34.3 \mathrm{GHz} / \mathrm{RIU}, 27.9 \mathrm{GHz} / \mathrm{RIU}$, and $38.4 \mathrm{GHz} / \mathrm{RIU}$, respectively. The sensing of analyte thickness using $\mathrm{FD}_{3}$ shows that the frequency shift greatly increases when the analyte thickness increases from 0 to $5 \mu \mathrm{m}$ and then slowly increases and reaches a saturated value at $12.5 \mu \mathrm{m}$, demonstrating that it is better suited to detect the thickness of thin layer analyte. The proposed asymmetric MM structures with tunable Fano resonances show potential applications as modulators, attenuators, filters, and sensors and can be readily applied in the $\mathrm{THz}$ band.

\section{REFERENCES}

1. Zhang R, Yang K, Abbasi QH, Qaraqe KA, Alomainy A. Analytical modelling of the effect of noise on the terahertz in-vivo communication channel for bodycentric nano-networks. Nano Commun Netw (2018) 15:59-68. doi:10.1016/j. nancom.2017.04.001

2. Augustin S, Frohmann S, Jung P, Hubers HW. Mask responses for single-pixel terahertz imaging. Sci Rep (2018) 8:1-17. doi:10.1038/s41598-018-23313-6

3. Jokubauskis D, Minkevicius L, Karaliunas M, Indrisiunas S, Kasalynas I, Raciukaitis $G$, et al. Fibonacci terahertz imaging by silicon diffractive optics. Opt Lett (2018) 43:2795-8. doi:10.1364/OL.43.002795

4. Gong A, Qiu Y, Chen X, Zhao Z, Xia L, Shao Y. Biomedical applications of terahertz technology. Appl Spectrosc Rev (2020) 55:418-38. doi:10.1080/ 05704928.2019.1670202

5. Yang X, Yang K, Zhao X, Lin Z, Liu Z, Luo S, et al. Terahertz spectroscopy for the isothermal detection of bacterial DNA by magnetic bead-based rolling circle amplification. Analyst (2017) 142:4661-9. doi:10.1039/c7an01438d

6. Chen T, Zhang Q, Li Z, Hu F. Intermolecular weak interactions of crystalline purine and uric acid investigated by terahertz spectroscopy and theoretical calculation. J Lumin (2020) 223:174-81. doi:10.1016/j.jlumin.2020.117198

7. Mernea M, Ulareanu R, Calboreanu O, Chiritoiu G, Cucu D, Mihailescu DF. $\mathrm{N}$-glycosylation state of TRPM8 protein revealed by terahertz spectroscopy and

\section{DATA AVAILABILITY STATEMENT}

The original contributions presented in the study are included in the article/Supplementary Material, and further inquiries can be directed to the corresponding author.

\section{AUTHOR CONTRIBUTIONS}

JQ conceived the idea and wrote part of the manuscript. YW performed the numerical simulations and analyzed the results. SJ prepared the figures and wrote part of the manuscript. All authors have given approval to the final version of the manuscript.

\section{ACKNOWLEDGMENTS}

The authors acknowledge the financial support from the National Natural Science Foundation of China (No. 61705213).

molecular modelling. Biochim Biophys Acta-Gen Subj (2020) 32:1864. doi:10. 1016/j.bbagen.2020.129580

8. Wu X, Wang L, Peng Y, Wu F, Cao J, Chen X, et al. Quantitative analysis of direct oral anticoagulant rivaroxaban by terahertz spectroscopy. Analyst (2020) 145:3909-15. doi:10.1039/d0an00268b

9. Zhou Q, Shen Y, Li Y, Xu L, Cai Y, Deng X. Terahertz spectroscopic characterizations and DFT calculations of carbamazepine cocrystals with nicotinamide, saccharin and fumaric acid. Spectroc Acta Pt A-Molec Biomolec Spectr (2020) 105:236. doi:10.1016/j.saa.2020.118346

10. Ornik J, Knoth D, Koch M, Keck CM. Terahertz-spectroscopy for nondestructive determination of crystallinity of L-tartaric acid in smartFilms (R) and tablets made from paper. Int. J Pharm (2020) 45:581. doi:10.1016/j. ijpharm.2020.119253

11. Chen L, Ren G, Liu L, Guo P, Wang E, Zhu Z, et al. Probing NaCl hydrate formation from aqueous solutions by terahertz time-domain spectroscopy. Phys Chem Chem Phys (2020) 22(32):17791-17797. doi:10.1039/d0cp01571g

12. Wang Y, Wang Q, Zhao Z, Liu A, Tian Y, Qin J. Rapid qualitative and quantitative analysis of chlortetracycline hydrochloride and tetracycline hydrochloride in environmental samples based on terahertz frequency-domain spectroscopy. Talanta (2018) 190:284-91. doi:10.1016/j.talanta.2018.08.008

13. Haxha S, AbdelMalek F, Ouerghi F, Charlton MDB, Aggoun A, Fang X. Metamaterial superlenses operating at visible wavelength for imaging applications. Sci Rep (2018) 8:10. doi:10.1038/s41598-018-33572-y 
14. Vendik IB, Vendik OG. Metamaterials and their application in microwaves: a review. Tech Phys (2013) 58:1-24. doi:10.1134/S1063784213010234

15. Watts CM, Liu X, Padilla WJ. Metamaterial electromagnetic wave absorbers. Adv Mater (2012) 24:OP98-OP120. doi:10.1002/adma.201200674

16. Tao H, Bingham CM, Strikwerda AC, Pilon D, Shrekenhamer D, Landy NI, et al. Highly flexible wide angle of incidence terahertz metamaterial absorber: design, fabrication, and characterization. Phys Rev B (2008) 15:78. doi:10.1103/ PhysRevB.78.241103

17. He Z, Wu L, Liu Y, Lu Y, Wang F, Shao W, et al. Ultrawide bandwidth and large-angle electromagnetic wave absorption based on triple-nested helix metamaterial absorbers. J Appl Phys (2020) 28:127. doi:10.1063/5.0001885

18. Zhong M, Jiang X, Zhu X, Zhang J, Zhong J. Design and preparation of a single-band tunable metamaterial absorber in terahertz frequency. Phys Scripta (2020) 125:95. doi:10.1088/1402-4896/ab7681

19. Islam SS, Faruque MRI, Islam MT. An object-independent ENZ metamaterialbased wideband electromagnetic cloak. Sci Rep (2016) 159:6. doi:10.1038/ srep33624

20. Cheng R, Xu L, Yu X, Zou L, Shen Y, Deng X. High-sensitivity biosensor for identification of protein based on terahertz Fano resonance metasurfaces. Optic Commun (2020) 56:473. doi:10.1016/j.optcom.2020.125850

21. Chen T, Li SY, Sun H. Metamaterials application in sensing. Sensors (2012) 12 : 2742-65. doi:10.3390/s120302742

22. Tao H, Kadlec EA, Strikwerda AC, Fan K, Padilla WJ, Averitt RD, et al. Microwave and Terahertz wave sensing with metamaterials. Optic Express (2011) 19:21620-6. doi:10.1364/OE.19.021620

23. Withayachumnankul W, Lin H, Serita K, Shah CM, Sriram S, Bhaskaran M, et al. Sub-diffraction thin-film sensing with planar terahertz metamaterials. Optic Express (2012) 20:3345-52. doi:10.1364/OE.20.003345

24. Wang W, Yan F, Tan S, Zhou H, Hou Y. Ultrasensitive terahertz metamaterial sensor based on vertical split ring resonators. Photon Res (2017) 5:571-7. doi:10.1364/PRJ.5.000571

25. Zhang C, Liang L, Ding L, Jin B, Hou Y, Li C, et al. Label-free measurements on cell apoptosis using a terahertz metamaterial-based biosensor. Appl Phys Lett (2016) 28:108. doi:10.1063/1.4954015

26. Zheng X, Zhao Z, Peng W, Zhao H, Zhang J, Luo Z, et al. Suppression of terahertz dipole oscillation in split-ring resonators deformed from square to triangle. Appl Phys Mater Sci Process (2017) 56:123. doi:10.1007/s00339-0170904-7

27. Gupta M, Singh R. Toroidal versus Fano resonances in high Q planar $\mathrm{THz}$ metamaterials. Adv Opt Mater (2016) 4(12):2119-25. doi:10.1002/adom. 201600553
28. Cong L, Manjappa M, Xu N, Al-Naib I, Zhang W, Singh R. Fano resonances in terahertz metasurfaces: a figure of merit optimization. Adv Opt Mater (2015) 3(11):1537-43. doi:10.1002/adom.201500207

29. Srivastava YK, Singh R. Impact of conductivity on Lorentzian and Fano resonant high-Q $\mathrm{THz}$ metamaterials: superconductor, metal and perfect electric conductor. J Appl Phys (2017) 64:122. doi:10.1063/1.4994951

30. Cao W, Singh R, Al-Naib IAI, He M, Taylor AJ, Zhang W. Low-loss ultra-highQ dark mode plasmonic Fano metamaterials. Opt Lett (2012) 37:3366-8. doi:10.1364/OL.37.003366

31. Wang B, Xie Z, Feng S, Zhang B, Zhang Y. Ultrahigh Q-factor and figure of merit Fano metamaterial based on dark ring magnetic mode. Optic Commun (2015) 335:60-4. doi:10.1016/j.optcom.2014.08.062

32. Singh R, Cao W, Al-Naib I, Cong L, Withayachumnankul W, Zhang W. Ultrasensitive terahertz sensing with high-Q Fano resonances in metasurfaces. Appl Phys Lett (2014) 73:105. doi:10.1063/1.4895595

33. Serita K, Murakami H, Kawayama I, Tonouchi M. A terahertz-microfluidic chip with a few arrays of asymmetric meta-atoms for the ultra-trace sensing of solutions. Photonics (2019) 56:6. doi:10.3390/photonics6010012

34. Park H-R, Chen X, Ngoc-Cuong N, Peraire J, Oh S-H. Nanogap-enhanced terahertz sensing of $1 \mathrm{~nm}$ thick (lambda/10(6)) dielectric films. ACS Photon (2015) 2(3):150204164400005. doi:10.1021/ph500464j

35. Srivastava YK, Ako RT, Gupta M, Bhaskaran M, Sriram S, Singh R. Terahertz sensing of $7 \mathrm{~nm}$ dielectric film with bound states in the continuum metasurfaces. Appl Phys Lett (2019) 115:151105. doi:10.1063/1.5110383

36. Zhao Z, Gu Z, Ako RT, Zhao H, Sriram S. Coherently controllable terahertz plasmon-induced transparency using a coupled Fano-Lorentzian metasurface. Optic Express (2020) 28:15573-86. doi:10.1364/OE.393714

37. Gu Z, Zhao Z, Zhao H, Peng W, Zhang J, Shi W. Fano-resonance collapse induced terahertz magnetic dipole oscillation in complementary meta-atoms via local symmetry breaking. J Appl Phys (2019) 46:125. doi:10.1063/1.5090153

Conflict of Interest: The authors declare that the research was conducted in the absence of any commercial or financial relationships that could be construed as a potential conflict of interest.

Copyright (C) 2021 Wang, Jia and Qin. This is an open-access article distributed under the terms of the Creative Commons Attribution License (CC BY). The use, distribution or reproduction in other forums is permitted, provided the original author(s) and the copyright owner(s) are credited and that the original publication in this journal is cited, in accordance with accepted academic practice. No use, distribution or reproduction is permitted which does not comply with these terms. 